\title{
The Time-to-Empty for Tandem Jackson Networks
}

\author{
Michael A. Zazanis \\ Department of Statistics \\ Athens University of Economics and Business \\ Patision 76, Athens 104 34, Greece \\ zazanis@aueb.gr tel: +302108203523
}

\begin{abstract}
We derive the distribution of the time-to-empty for an open tandem Jackson network assuming that, while in equilibrium at time 0 , the arrival stream is suddenly shut off. The analysis is based on analogous results regarding the distribution of the time-to-empty for the corresponding closed tandem Jackson network. The results obtained are used in the analysis of a two-class tandem Jackson network with FIFO discipline where customers of the second class have negligible service times.
\end{abstract}

Keywords: JACKSON Networks, TIME TO EMPTy, CyClic NeTWORKS

SHORT TITLE: TIME-TO-EMPTY FOR TANDEM NETWORKS

\section{Introduction}

We consider a open tandem Jackson network with $M$ single server exponential stations with service rates $\mu_{m}, m=1,2, \ldots, M$, and external Poisson arrivals with rate $\lambda<\min _{m=1, \ldots, M} \mu_{m}$. Assuming the process to be in equilibrium, we are interested in the distribution of the time it takes for the system to empty if at time $t=0$ the arrival process is suddenly switched off. 
More formally consider the continuous time Markov chain $\mathbf{X}=\{\mathbf{X}(t) ; t \in \mathbb{R}\}$ with state space $\mathscr{S}:=\left\{\mathbf{n}:=\left(n_{1}, \ldots, n_{M}\right), n_{m} \in \mathbb{N}\right\}$ and generator

$$
\begin{aligned}
q\left(\mathbf{n}, \mathbf{n}+\mathbf{e}_{m+1}-\mathbf{e}_{m}\right) & =\mu_{m} \mathbf{1}\left(n_{m}>0\right), \quad m=1,2, \ldots, M-1, \\
q\left(\mathbf{n}, \mathbf{n}-\mathbf{e}_{M}\right) & =\mu_{M} \mathbf{1}\left(n_{M}>0\right), \\
q(\mathbf{n}, \mathbf{n}) & =-\sum_{m=1}^{M} \mu_{m} \mathbf{1}\left(n_{m}>0\right) \\
q\left(\mathbf{n}, \mathbf{n}^{\prime}\right) & =0, \quad \text { for any other } \mathbf{n}^{\prime} .
\end{aligned}
$$

(Here $\mathbf{e}_{m}=(0,0, \ldots, 1, \ldots, 0)$ is the unit vector in the $m$ th direction in $\mathbb{R}^{M}$ and $(0,0, \ldots, 0)$ acts as an absorbing state.) The above process, together with the initial condition $P(\mathbf{X}(0)=$ $\mathbf{n})=\prod_{m=1}^{M}\left(1-\rho_{m}\right) \rho_{m}^{n_{m}}$, describes the behavior of the open tandem Jackson network when the arrival process is switched off at time $t=0$.

Note that the time-to-empty is precisely the flow time through the network of a virtual customer who arrives at time $t=0$ and finds the system in equilibrium, and whose service time is zero at all stations. Thus, the time-to-empty can be thought of as a generalization of the workload (or virtual waiting time) for a tandem network. In section 4 we take advantage of this remark to apply the results obtained to the analysis of a two-class FIFO tandem network where customers of the second class have negligible service times.

Let $\tau=\inf \{t \geq 0: \mathbf{X}(t)=(0,0, \ldots, 0)\}$ be the time required for the system to empty. In order to compute the Laplace transform $R(s):=E e^{-s \tau}$ we first condition on the number of customers in the network at time 0 . If we denote by

$$
R_{N}(s):=E\left[e^{-s \tau} \mid \sum_{m=1}^{M} X_{m}(0)=N\right]
$$

the Laplace transform of $\tau$ conditional on having $N$ customers in the system initially, then

$$
R(s)=\sum_{N=0}^{\infty} R_{N}(s) P\left(\sum_{m=1}^{M} X_{m}(0)=N\right) .
$$

The total number of customers in the system in steady state for the original open Jackson network, before the arrival process is switched off, can of course be obtained as the sum of $M$ 
independent geometric random variables and thus its probability generating function takes the form

$$
\sum_{N=0}^{\infty} z^{N} P\left(\sum_{m=1}^{M} X_{m}(0)=N\right)=\prod_{m=1}^{M} \frac{1-\rho_{m}}{1-\rho_{m} z}=\sum_{m=1}^{M} c_{m} \frac{1-\rho_{m}}{1-\rho_{m} z},
$$

where the constants

$$
c_{m}=\prod_{l \neq m} \frac{1-\rho_{l}}{1-\rho_{l} / \rho_{m}}, \quad m=1,2, \ldots, M,
$$

are determined by a partial fractions expansion. The above expansion is of course valid only if the service rates are different from each other i.e. $\mu_{m} \neq \mu_{l}$ (and thus $\rho_{m} \neq \rho_{l}$ ) for $m \neq l$, an assumption which we will adopt for simplicity in the sequel. Thus we have

$$
P\left(\sum_{m=1}^{M} X_{m}(0)=N\right)=\sum_{m=1}^{M} c_{m}\left(1-\rho_{m}\right) \rho_{m}^{N}, \quad N=0,1,2, \ldots
$$

In order to evaluate $R_{N}(s)$ it suffices to realize that this is the same as the time to emptiness for the corresponding closed Jackson network in equilibrium if at time 0 we "break the loop" and suppose that customers who finish service at station $M$ simply leave the system and do not return to station 1 . In the next section we will see how this can be evaluated by turning what is a question regarding the transient behavior of the network into a question regarding its steady-state operation.

\section{A recursive relationship for the time-to-empty in tandem closed networks}

Consider the same tandem network as before which we now assume operating as a closed Jackson network with $N$ customers. In fact we will consider a whole family of closed network processes, each described by a continuous time Markov Chain, $\mathbf{X}^{N}=\left\{\mathbf{X}^{N}(t) ; t \in \mathbb{R}\right\}$ with state space $\mathscr{S}:=\left\{\mathbf{n}:=\left(n_{1}, \ldots, n_{M}\right), n_{m} \in \mathbb{N}, \sum_{m=1}^{M} n_{m}=N\right\}$ defined on a probability space $(\Omega, \mathscr{F}, P)$, where $\mathbf{X}^{N}(t)=\left(X_{1}^{N}(t), \ldots, X_{M}^{N}(t)\right)$ denotes the number of customers in each station at time $t$. We assume that the Markov Chain $\mathbf{X}^{N}$ is stationary under $P$ and we 
denote by $\left\{D_{n}^{N}\right\}$ the point process of departures from node $M$. We use the standard numbering convention that $D_{0}^{N} \leq 0<D_{1}^{N} P$-a.s. (i.e. call $D_{1}^{N}$ the first point strictly to the right of the time origin) and denote by $d_{n}^{N}:=D_{n+1}^{N}-D_{n}^{N}, n \in \mathbb{Z}$, the interdeparture times. We will also denote by $P_{N}^{0}$ the Palm transformation of the probability measure $P$ under the point process $\left\{D_{n}^{N}\right\}$ and by $E_{N}^{0}$ expectation with respect to $P_{N}^{0}$.

If we were to assume that at time 0 "the loop is broken" between station $M$ and station 1 so that customers who finish service at station $M$ simply leave the system without returning then, since at time 0 the system is a closed network in equilibrium, the time for the system to empty is precisely $D_{N}^{N}$, the time till the $N$ th departure in a closed network with $N$ customers in equilibrium. This of course is true since all stations have single servers so that it is not possible for one customer to overtake another. Thus

$$
R_{N}(s)=E e^{-s D_{N}^{N}}
$$

We are now ready to establish the following

Theorem 1. The time-to-empty starting from equilibrium for a closed tandem Jackson network with $N$ customers is given by the expression

$$
R_{N}(s)=(-1)^{N-1} \frac{\lambda_{N} \lambda_{N-1} \cdots \lambda_{2}}{(N-1) !} \frac{d^{N-1}}{d s^{N-1}} R_{1}(s)
$$

where $\lambda_{k}$ is the throughput in such a network with $k$ customers and $R_{1}(s)$ is the time to emptiness from equilibrium in the network with a single customer given by

$$
R_{1}(s)=\lambda_{1} \frac{1-E_{1}^{0}\left[e^{-s D_{1}^{1}}\right]}{s}
$$

where

$$
E_{1}^{0}\left[e^{-s D_{1}^{1}}\right]=\prod_{m=1}^{M} \frac{\mu_{m}}{\mu_{m}+s} .
$$


Proof: We will use the approach in [10] (see also [12]) as follows. Let $\mathbf{X}^{N+1}$ be the same closed Jackson network with $N+1$ customers and suppose that it is stationary under $P$. If we denote by $\left\{D_{n}^{N+1}\right\}$ the departure process for this system and if $P_{N+1}^{0}$ denotes the Palm probability transformation of $P$ under $\left\{D_{n}^{N+1}\right\}$ then, as a consequence of the arrival theorem (see [6], [8], [3], [9], [7]), we have

$$
R_{N}(s)=E_{N+1}^{0} e^{-s D_{N}^{N+1}} .
$$

However, by the shift invariance properties of $P_{N+1}^{0}$, and since $P_{N+1}^{0}\left(D_{0}^{N+1}=0\right)=1$, we also have

$$
R_{N}(s)=E_{N+1}^{0} e^{-s\left(D_{N}^{N+1}-D_{0}^{N+1}\right)}=E_{N+1}^{0} e^{-s\left(D_{N-1}^{N+1}-D_{-1}^{N+1}\right)}=\cdots=E_{N+1}^{0} e^{-s\left(D_{1}^{N+1}-D_{-N+1}^{N+1}\right)} .
$$

Using the above equations in conjunction with the arrival theorem we conclude that

$$
R_{N}(s)=E_{N+1}^{0} e^{-s\left(D_{N-k}^{N+1}-D_{-k}^{N+1}\right)}=E e^{-s\left(D_{N-k}^{N}-D_{-k+1}^{N}\right)}, \quad k=1,2, \ldots, N-1 .
$$

(In the above argument note that $R_{N}(s)$ is originally expressed as a stationary expectation, then the arrival theorem is used to express it as a Palm expectation in a network with one additional customer, then a shift is performed along the points of the departure process, and finally the arrival theorem is used once more to return to a stationary expectation in the original network with $N$ customers.) Using the Palm inversion formula (see [1]) we obtain the relationship

$$
\begin{aligned}
E e^{-s\left(D_{N-k}^{N}-D_{-k+1}^{N}\right)} & =\lambda_{N} E_{N}^{0} \int_{0}^{D_{1}^{N}} e^{-s\left(D_{N-k}^{N}-D_{-k+1}^{N}\right)} d t=\lambda_{N} E_{N}^{0}\left[d_{0}^{N} e^{-s\left(D_{N-k}^{N}-D_{-k+1}^{N}\right)}\right] \\
& =\lambda_{N} E_{N}^{0}\left[d_{k-1}^{N} e^{-s\left(D_{N-1}^{N}-D_{0}^{N}\right)}\right], \quad k=1,2, \ldots, N-1
\end{aligned}
$$

where, in the last equation we have again used the shift invariance of $P_{N}^{0}$. From (8) and (9), adding term by term, we obtain

$$
(N-1) R_{N}(s)=\sum_{k=0}^{N-2} \lambda_{N} E_{N}^{0}\left[d_{k}^{N} e^{-s\left(D_{N-1}^{N}-D_{0}^{N}\right)}\right]=\lambda_{N} E^{0}\left[D_{N-1}^{N} e^{-s D_{N-1}^{N}}\right],
$$

where, in the above equation we have taken into account that $P_{N}^{0}$ a.s. $D_{0}^{N}=0$ and $D_{N-1}^{N}=$ $d_{0}^{N}+d_{1}^{N}+\cdots+d_{N-2}^{N}$. However we have

$$
E^{0}\left[D_{N-1}^{N} e^{-s D_{N-1}^{N}}\right]=-\frac{d}{d s} E_{N}^{0}\left[e^{-s D_{N-1}^{N}}\right]=-\frac{d}{d s} E\left[e^{-s D_{N-1}^{N-1}}\right]=-\frac{d}{d s} R_{N-1}(s)
$$


(where we have used the arrival theorem again!) and hence equation (10) can be written as

$$
R_{N}(s)=-\frac{\lambda_{N}}{N-1} \frac{d}{d s} R_{N-1}(s)
$$

Applying the above recursively we obtain readily (5). It remains to establish (6) which however follows immediately by applying the Palm inversion formula once more. (This should be intuitively obvious: $R_{1}(s)=E\left[e^{-s D_{1}^{1}}\right]$ is simply the forward recurrence time of the departure process $\left\{D_{n}^{1}\right\}$ in the closed network with a single customer in equilibrium.) Finally, (7) clearly holds since $D_{1}^{1}$ under $P_{1}^{0}$ is the typical cycle time in a closed tandem network with a single customer.

From the above theorem it is clear that the explicit computation of $R_{N}(s)$ is not difficult. In the next section we carry out this computation and we return to the problem of the open network.

\section{Closed form expressions for the time-to-empty in closed and open Jackson networks}

Here we will assume for simplicity that the service rates in different nodes of the network are different $\left(\mu_{m} \neq \mu_{l}\right.$ when $\left.\mu \neq l\right)$. Under this assumption it has been shown in [10] that

$$
\lambda_{k}=\frac{\sum_{m=1}^{M} \alpha_{m} \mu_{m}^{-k+1}}{\sum_{m=1}^{M} \alpha_{m} \mu_{m}^{-k}}
$$

where

$$
\alpha_{m}=\prod_{l \neq m} \frac{\mu_{l}}{\mu_{l}-\mu_{m}}, \quad m=1,2, \ldots, M .
$$

The constants $\alpha_{m}$ arise from the partial fractions expansion

$$
\prod_{m=1}^{M} \frac{\mu_{m}}{\mu_{m}+s}=\sum_{m=1}^{M} \alpha_{m} \frac{\mu_{m}}{\mu_{m}+s} .
$$


If we set $C:=\prod_{m=1}^{M}\left(1-\rho_{m}\right)$ then it is easy to see that these constants are connected to the constants (3) via the relationship

$$
c_{m}=\frac{C}{1-\rho_{m}} \alpha_{m}
$$

From (5), (6), (7), (12), and (14), after some elementary manipulations, we obtain the following closed form expression for the time-to-empty for a closed tandem Jackson network initially in equilibrium with $N$ customers:

$$
R_{N}(s)=\frac{\sum_{m=1}^{M} \alpha_{m}\left(\mu_{m}+s\right)^{-N}}{\sum_{m=1}^{M} \alpha_{m} \mu_{m}^{-N}} .
$$

It is interesting to compare the above formula with the expression for the Laplace transform of the cycle time of a customer in this network with $N$ customers, which we will denote by $\Phi_{N}(s)$, as given in [10]

$$
\Phi_{N}(s)=\frac{\sum_{m=1}^{M} \alpha_{m} \mu_{m}\left(\mu_{m}+s\right)^{-N}}{\sum_{m=1}^{M} \alpha_{m} \mu_{m}^{-N+1}}
$$

We are now in a position to compute the Laplace transform of the time to empty for the open tandem Jackson network in steady state. From (1), (4), (15), and (16) we obtain

$$
\begin{aligned}
R(s) & =C \sum_{N=0}^{\infty} \sum_{m=1}^{M} \alpha_{m} \rho_{m}^{N} \frac{\sum_{m=1}^{M} \alpha_{m}\left(\mu_{m}+s\right)^{-N}}{\sum_{m=1}^{M} \alpha_{m} \mu_{m}^{-N}} \\
& =\prod_{m=1}^{M}\left(1-\rho_{m}\right)+\sum_{m=1}^{M} \rho_{m}\left(\prod_{l \neq m} \frac{1-\rho_{l}}{1-\rho_{l} / \rho_{m}}\right) \frac{\mu_{m}-\lambda}{\mu_{m}-\lambda+s} .
\end{aligned}
$$

Again, the comparison with the corresponding flow time, namely the time it takes from the moment a customer arrives to the first station till the time it leaves the last station in steady state is interesting. If this flow time is denoted by $\Phi(s)$, then it is well known (e.g. see [9], [7]) that

$$
\Phi(s)=\prod_{m=1}^{M} \frac{\mu_{m}-\lambda}{\mu_{m}-\lambda+s}=\sum_{m=1}^{M}\left(\prod_{l \neq m} \frac{1-\rho_{l}}{1-\rho_{l} / \rho_{m}}\right) \frac{\mu_{m}-\lambda}{\mu_{m}-\lambda+s},
$$

the second expression above following by a partial fractions expansion of the first. 
Finally we compute the Laplace transform of the time-to-empty for the same system assuming that at time 0 it is in steady state and conditioning on the event that there are $N$ or more customers in the system. Elementary manipulations similar to the ones described above give

$$
E\left[e^{-s \tau} \mid \sum_{m=1}^{M} X_{m}(0) \geq N\right]=\frac{\sum_{m=1}^{M} c_{m} \rho_{m}^{N}\left(\frac{\mu_{m}}{\mu_{m}+s}\right)^{N}\left(1-\rho_{m}+\rho_{m} \frac{\mu_{m}-\lambda}{\mu_{m}-\lambda+s}\right)}{\sum_{m=1}^{M} c_{m} \rho_{m}^{N}},
$$

where the constants $c_{m}$ are given in (3). Note the similarity of the above expression with the corresponding result for the $M / M / 1$ queue. Suppose that an M/M/1 system with arrival rate $\lambda$ and service rate $\mu>\lambda$ is in equilibrium at time $t=0$, and at that time arrival process is switched off. Then the Laplace transform of the time required for the system to empty can be easily computed via a conditioning argument and is equal to

$$
\left(\frac{\mu}{\mu+s}\right)^{N}\left(1-\rho+\rho \frac{\mu-\lambda}{\mu-\lambda+s}\right)
$$

where $\rho=\lambda / \mu$. It is worth noting that the corresponding result for the tandem network given in (20) is simply a weighted average of the corresponding $M / M / 1$ factors for each station.

From (20) we can readily obtain the mean time for the network to empty given that there are at least $N$ customers in the system

$$
E\left[\tau \mid \sum_{m=1}^{M} X_{m}(0) \geq N\right]=N \frac{\sum_{m=1}^{M} c_{m} \rho_{m}^{N} \mu_{m}^{-1}}{\sum_{m=1}^{M} c_{m} \rho_{m}^{N}}+\frac{\sum_{m=1}^{M} c_{m} \rho_{m}^{N+1}\left(\mu_{m}-\lambda\right)^{-1}}{\sum_{m=1}^{M} c_{m} \rho_{m}^{N}} .
$$

\section{Application: A two-class tandem network with probe cus- tomers}

Consider now an open, tandem Jackson network with two classes of customers. Customers belonging to the first class arrive to the system according to a Poisson process with rate $\lambda$ and their service rates at the $M$ stations are $\mu_{1}, \mu_{2}, \ldots, \mu_{M}$, as before. Customers belonging to the second class, arrive according to an independent Poisson process with rate $\nu$ and the 
service discipline is FIFO for all customers and stations. We will assume that the service times of class- 2 customers at the various stations are so small compared with the time scale of the system that we can entirely ignore them. Thus these customers, which we will call probe customers, flow through the system without delay except for the fact that they have to wait in front of class-1 customers. There are many cases both in communications systems and in manufacturing where such a model is applicable. Then one can easily see that the flow time of the typical probe customer is precisely the time-to-empty for the Jackson network in equilibrium with only one class of customers. Thus (18) gives also the flow time for probe customers. Let us now denote by $\Pi(z)$ the probability generating function (p.g.f.) of the stationary number of probe customers in the system. Then we can use the distributional law of Little (see [4], [5]) to obtain

$\Pi(z)=R(\nu(1-z))=\prod_{m=1}^{M}\left(1-\rho_{m}\right)+\sum_{m=1}^{M} \rho_{m}\left(\prod_{l \neq m} \frac{1-\rho_{l}}{1-\rho_{l} / \rho_{m}}\right) \frac{\mu_{m}-\lambda}{\mu_{m}-\lambda+\nu-\nu z}$.

Thus the stationary distribution of probe customers is expressed as a combination of geometric factors.

One might be tempted to derive directly $\Pi(z)$ by noting that the number of probe customers who arrive between two consecutive class-1 customers is geometrically distributed with p.g.f. given by $\frac{\lambda}{\lambda+\nu-\nu z}$ and then composing this p.g.f. with the p.g.f. of the total number of class-1 customers in the system, as given in (2). Such reasoning, however, will not lead to the correct result since the number of class- 1 customers in the system at any given time and the interarrival intervals corresponding to these customers are, of course, not independent.

Clearly, as long as the process of class- 1 customers is in steady state, the flow time of probe customers will be given by (18), regardless of the statistics of their arrival process, provided of course that this arrival process be independent of the arrivals and service requirements of class- 1 customers. What may become more complicated is the determination of the statistics of the total number of probe customers in the network. However, when probe customers 
arrive according to a renewal process with interarrival distribution $G$, independent of the arrivals and service times of class- 1 customers, then, as we will see, the stationary number of probe customers in the system can be easily determined. This is done using the distributional version of Little's law for renewal arrivals in the form given in [11] (see also [2]).

In the framework adopted there, one starts with a marked point process $\left\{\left(T_{n}, W_{n}\right) ; n \in\right.$ $\mathbb{Z}\}$, which is assumed to be stationary under the probability measure $P$. The marks $W_{n}$ are assumed to be non-negative w.p. 1. Corresponding to this marked point process one defines the arrival counting measure $A(B):=\sum_{n \in \mathbb{Z}} \mathbf{1}\left(T_{n} \in B\right)$, where $B$ is any Borel set in $\mathbb{R}$, and the system size process $Y(t):=\sum_{n \in \mathbb{Z}} \mathbf{1}\left(T_{n} \leq t<T_{n}+W_{n}\right) . T_{n}$ is to be interpreted as the arrival epoch of the $n$th customer, $W_{n}$ as his sojourn time through the system, and $Y(t)$ as the total number of customers in the system at time $t$. In our situation, $\left\{T_{n}\right\}$ is the renewal arrival process of probe customers, $\left\{W_{n}\right\}$ their corresponding sojourn times through the network, and $Y(t)$ the total number of probe customers in the network at time $t$. We will also denote by $P^{*}$ the Palm transformation of $P$ with respect to the point process $\left\{T_{n}\right\}$. Let $\Pi(z):=E z^{Y(0)}$ denote the p.g.f. of the stationary number of probe customers in the system. In [11] it is shown that

$$
\Pi(z)=1-\nu(1-z) \int_{0}^{\infty} P^{*}\left(W_{0}>u\right) E^{*}\left[z^{A(0, u]}\right] d u,
$$

where $\nu^{-1}=\int_{0}^{\infty} u d G(u)$ is the mean interarrival time, provided that the following two conditions hold:

a) The system is FIFO, i.e. $T_{m}+W_{m} \leq T_{n}+W_{n}$ w.p. 1 whenever $m<n$.

b) The system enjoys the following Lack of Anticipation Property: Under $P^{*}, W_{0}$ and $A(0, u]$ are independent for all $u>0$. This simply states that the sojourn time of a customer cannot be influenced by future arrivals, and future arrivals in turn do not contain any information regarding the sojourn time of the customer who arrives at 0 . 
In our situation it is clear that both conditions are satisfied. Also, $E^{*}\left[z^{A(0, u]}\right]=\sum_{n=0}^{\infty} z^{n} P^{*}\left(T_{n} \leq\right.$ $\left.u<T_{n+1}\right)$ and, if we denote by $\widehat{G}(s)=\int_{0}^{\infty} e^{-s t} d G(t)$ the Laplace transform of $G$, a straightforward computation gives

$$
\nu \int_{0}^{\infty} e^{-s u} E^{*}\left[z^{A(0, u]}\right] d u=\frac{\widehat{G}_{I}(s)}{1-z \widehat{G}(s)}
$$

where we have used the notation $\widehat{G}_{I}(s):=\nu s^{-1}(1-\widehat{G}(s))$ to denote the Laplace transform of the integrated tail distribution that corresponds to $G$. In view of (18) and (3) we have

$$
P^{*}\left(W_{0}>u\right)=\sum_{m=1}^{M} c_{m} \rho_{m} e^{-u\left(\mu_{m}-\lambda\right)} .
$$

Thus, from (22), (23), and (24) we conclude that

$$
\Pi(z)=1-\sum_{m=1}^{M} c_{m} \rho_{m} \frac{(1-z) \widehat{G}_{I}\left(\mu_{m}-\lambda\right)}{1-z \widehat{G}\left(\mu_{m}-\lambda\right)} .
$$

The above displays the total number of probe customers in the system as a combination of geometric factors.

\section{References}

[1] Baccelli, F., and P. Brémaud (1994). Elements of Queueing, Springer-Verlag, New York.

[2] Bertsimas, D. and D. Nakazato, (1995). “The distributional Little's Law and its applications," Oper. Res., 43, 298-310.

[3] Brémaud, P., R. Kanurpatti, and R. Mazumdar (1992). Event and time averages: a review. Adv. Appl. Prob. 24, 377-411.

[4] Haji, R. and G.F. Newell (1971). "A relation between stationary queue and waiting-time distributions," J. Appl. Prob., 8, 617-620.

[5] Keilson, J., and L.D. Servi (1988). "A distributional form of Little's law," Oper. Res. Letters, 7, 223-227. 
[6] Lavenerg, S.S. and M. Reiser (1980). Stationary state probabilities at arrival instants for closed queueing networks with multiple types of customers. J. Appl. Prob. 17, 10481061.

[7] Serfozo, R. F. (1999). Introduction to Stochastic Networks. Springer, New York.

[8] Sevcik, K.C. and I. Mitrani (1981). The distribution of queueing network states at input and output instants. J. Assoc. Comput. Mach. 28, 358-371.

[9] Walrand, J. (1988). An Introduction to Queueing Networks, Prentice-Hall.

[10] Zazanis, M.A. (1997). Formulas and representations for cyclic markovian networks via Palm calculus, Queueing Systems, 26, 1-2, 151-167.

[11] Zazanis, M.A. (1998). Functional Versions of Little's Law, Stochastic Processes and their Applications, 74, 2, 195-201.

[12] Zazanis M.A. (2003). Cycle Times in Single Server Cyclic Jackson Networks, technical report, Athens University of Economics and Business. 